\title{
Revised Point of Departure Design Options for Nuclear ThermalfPropulsion
}

\author{
James E. Fittje ${ }^{1}$ \\ Bruce G. Schnitzler² \\ Stanley Borowoski ${ }^{3}$
}

${ }_{1}^{1}$ Vantage Partners,(216) 416-5565, James.E.Fittje@grc.nasa.gov

2 Oak Ridge National Laboratory

${ }^{3}$ NASA Glenn Research Center 


\section{Introduction}

- Brief NTR Systems Background

- Fuel Element Geometries

- MCNP/NESS Model

- NERVA Derived Designs

- Criticality Limited

- 111 kN (25 klb f $_{f}$ Thrust Class

- Cermet Based Designs

- Criticality Limited

- 111 kN (25 klb f $_{f}$ Thrust Class

- Analysis Results

- Questions 


\section{Typical NTR System}

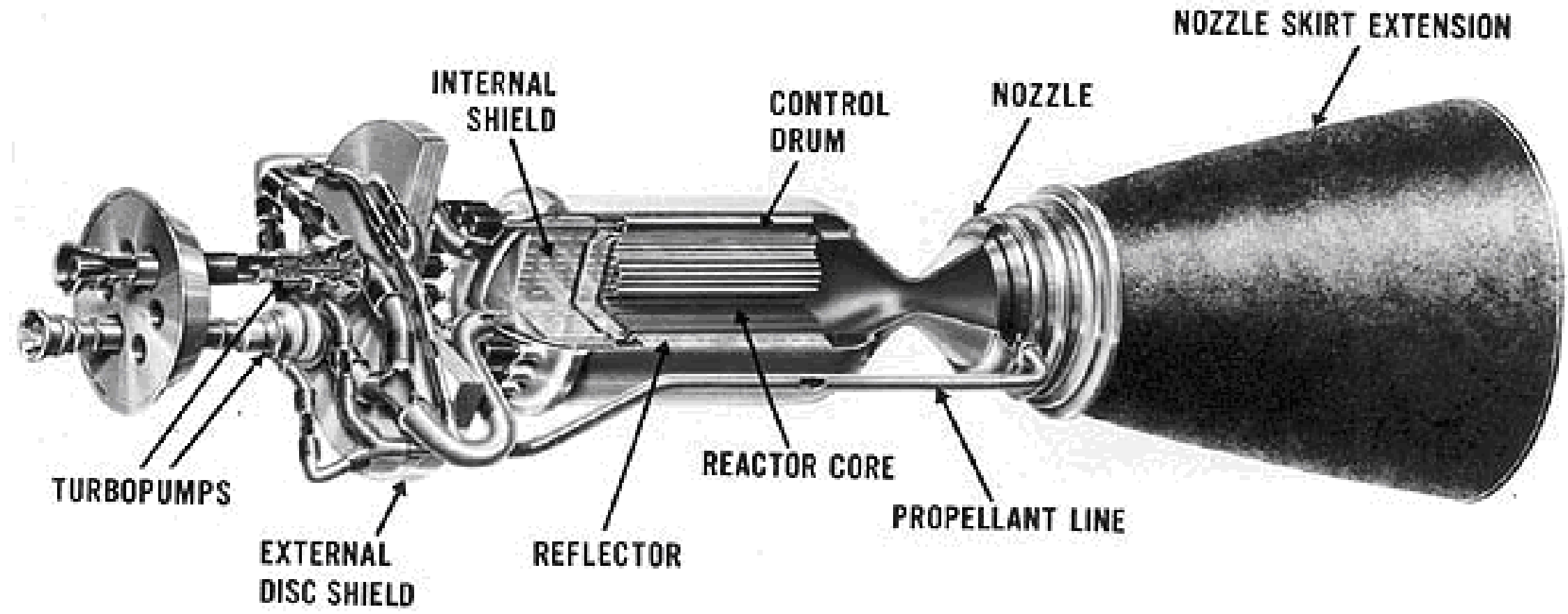

Glenn Research Center 


\section{Typical NTR Core Cross Section}

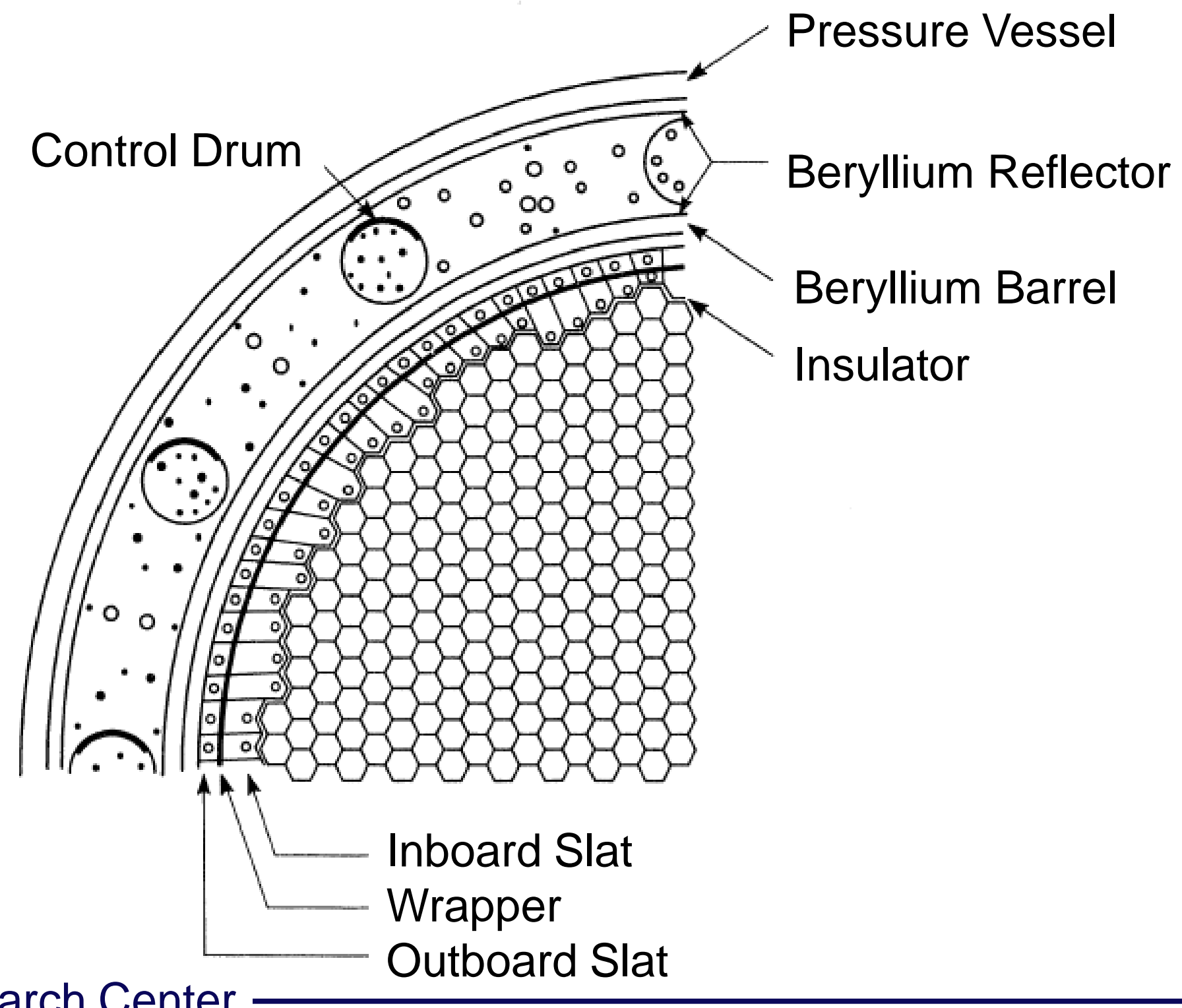




\section{NERVA Derived Reactor Cross Section}

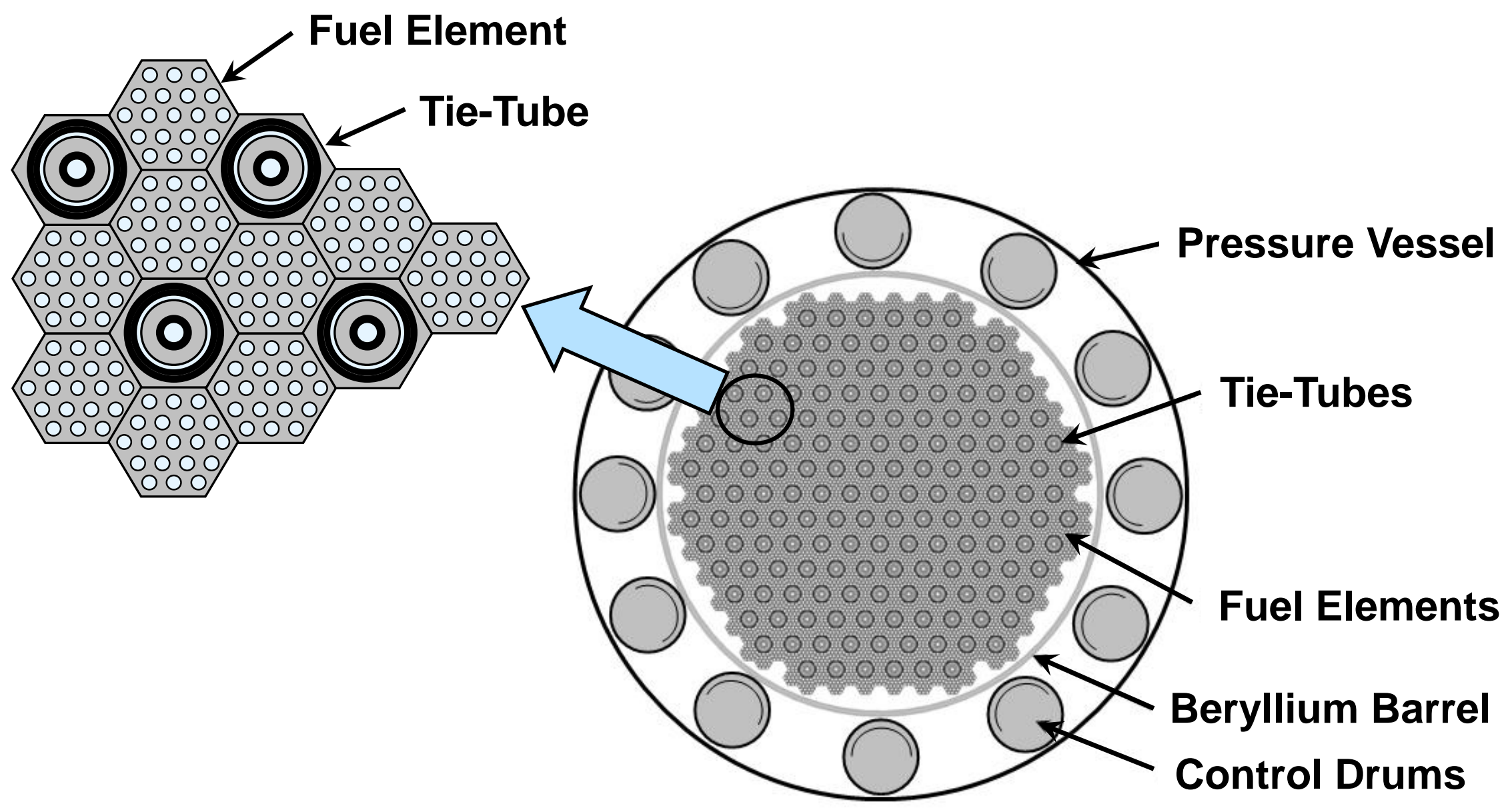

Glenn Research Center 


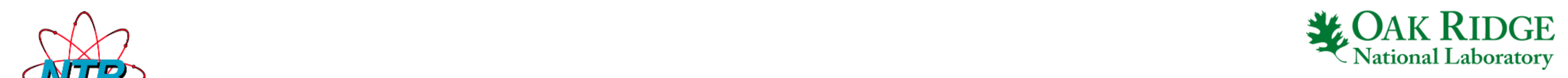

\section{Fuel Element and Tie Tube Arrangement}

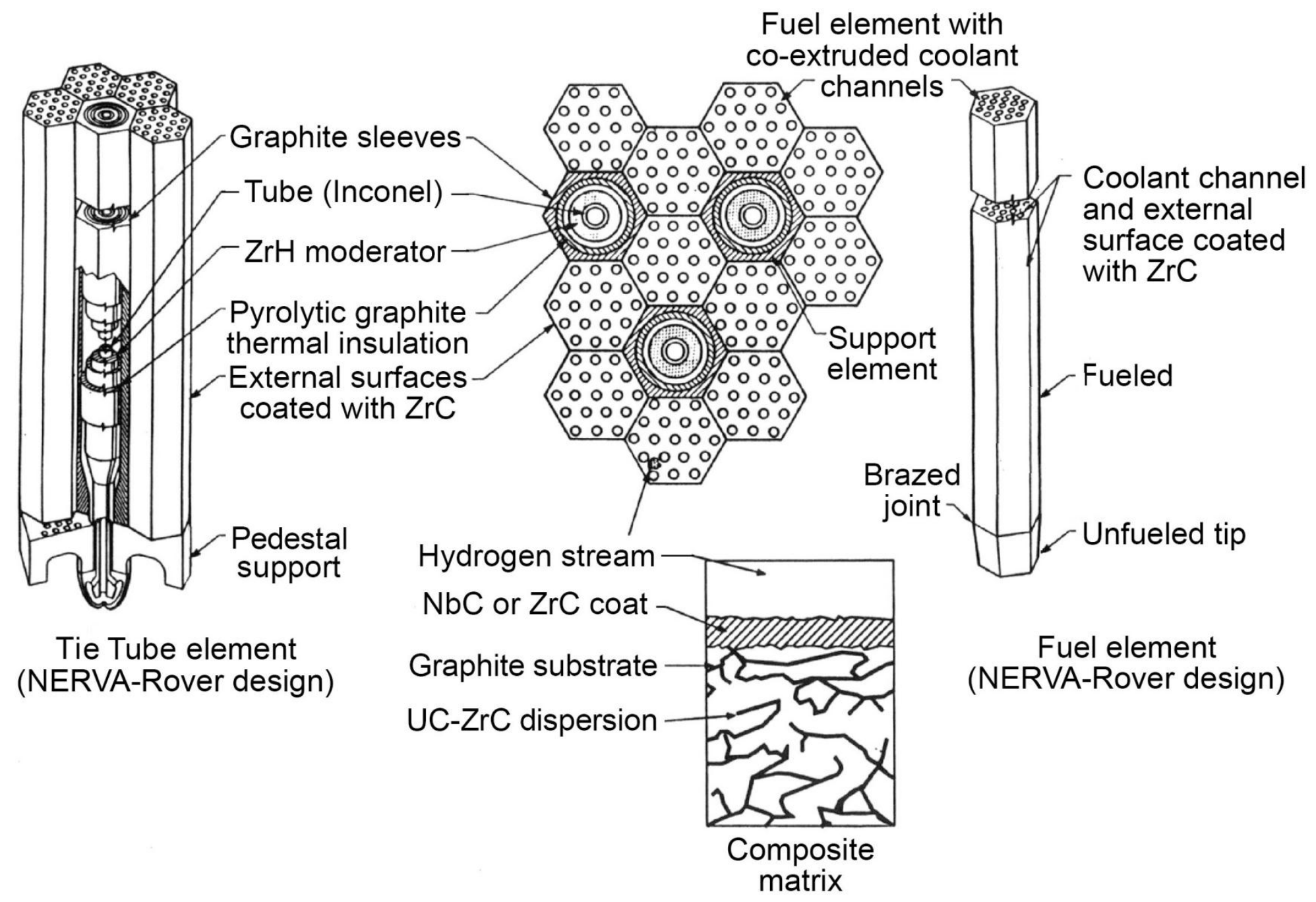




\section{Fuel Element And Tie Tube Cross Sections}

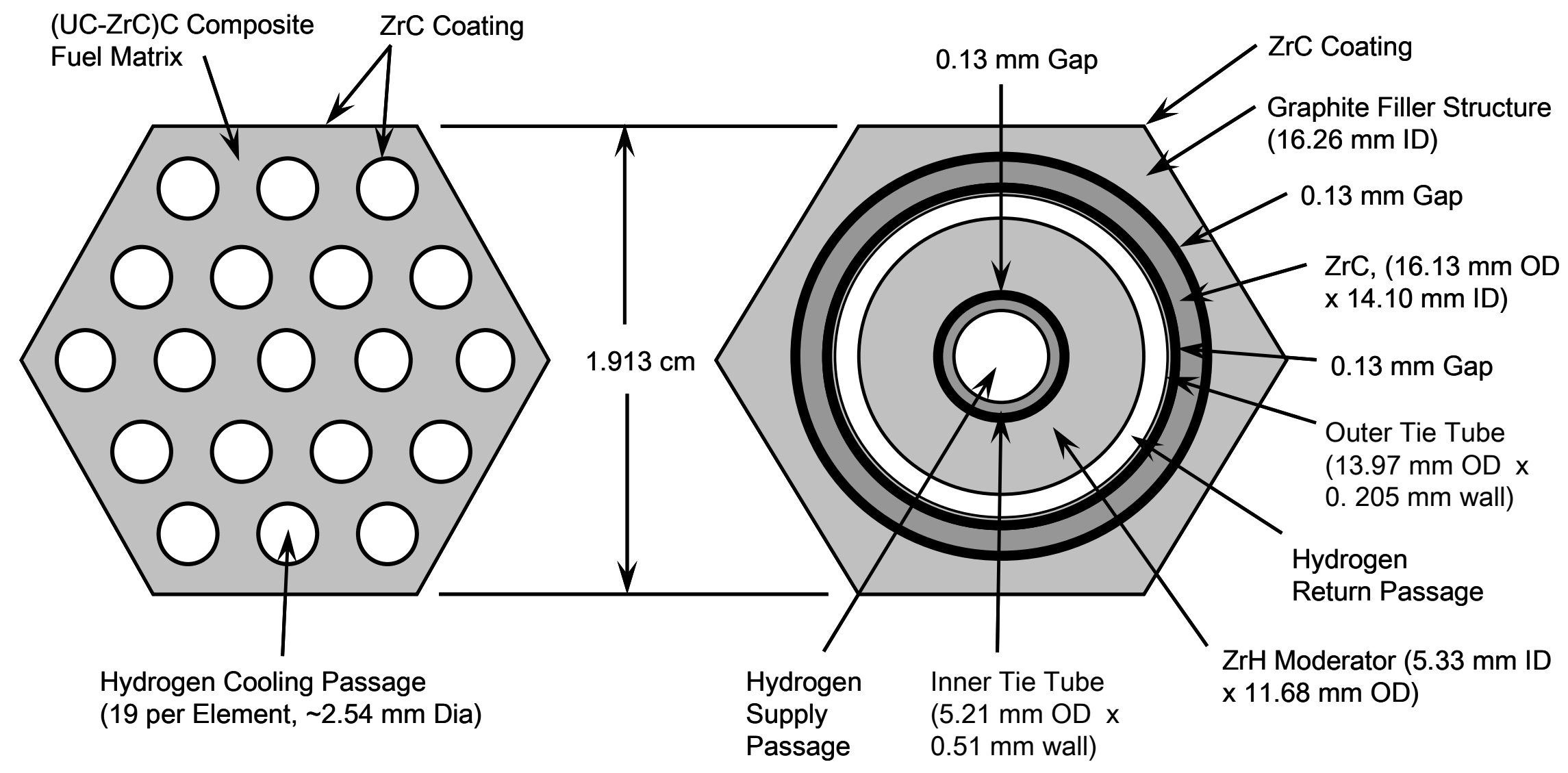




\section{GE-710 and GE-711 Comparison}

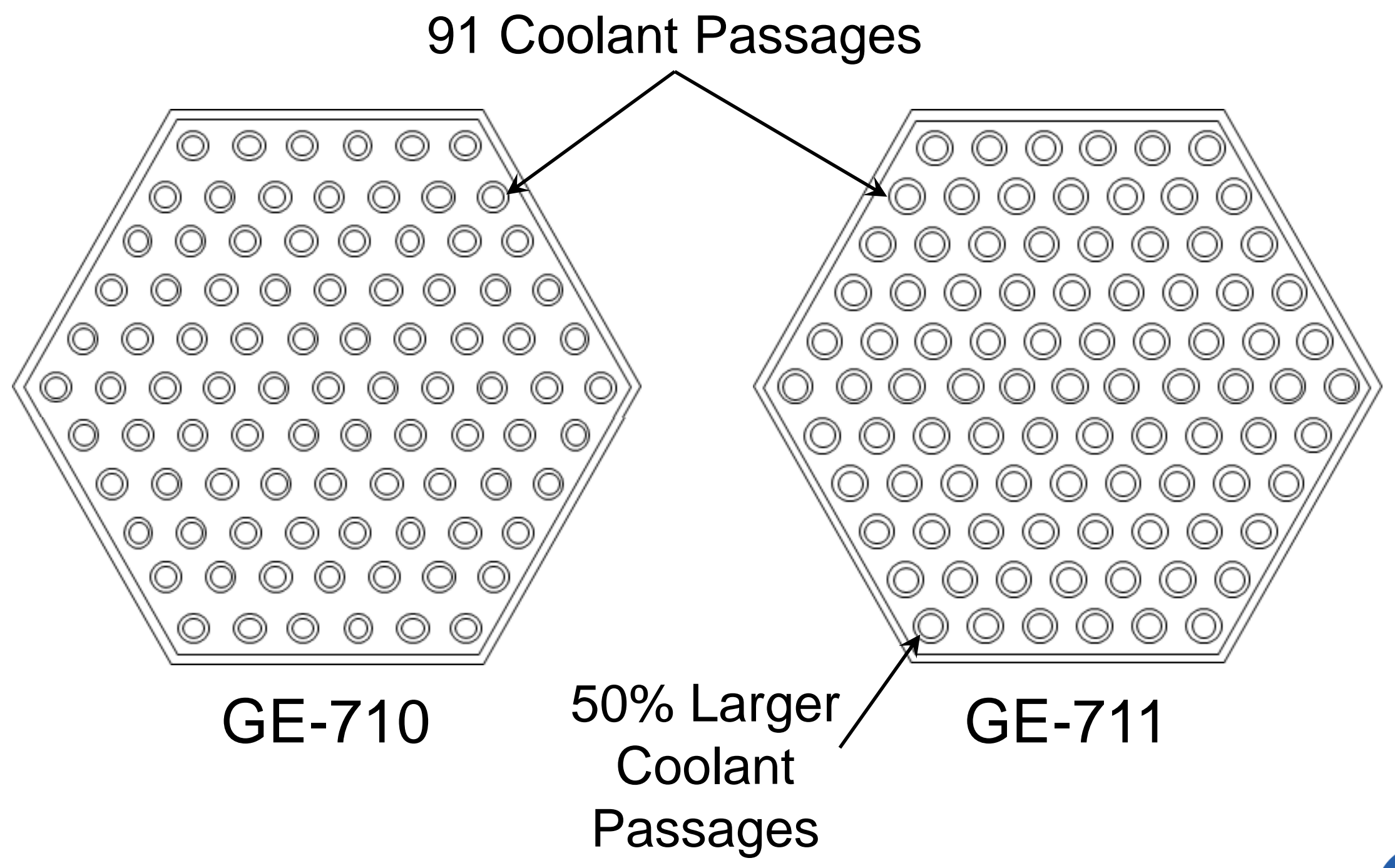




\section{GE-710 and GE-711 Comparison, cont.}

\begin{tabular}{|c|c|c|c|c|}
\hline Parameter & \multicolumn{2}{|c|}{ GE-710 } & \multicolumn{2}{|c|}{ GE-711 } \\
\hline $\begin{array}{l}\text { Fuel Element Width w/o } \\
\text { Cladding }\end{array}$ & $2.278 \mathrm{~cm}$ & 0.8976 in & $2.278 \mathrm{~cm}$ & 0.8976 in \\
\hline $\begin{array}{c}\text { Fuel Element Width with } \\
\text { Cladding }\end{array}$ & $2.361 \mathrm{~cm}$ & 0.9296 in & $2.361 \mathrm{~cm}$ & 0.9296 in \\
\hline Outer Cladding Thickness & $0.381 \mathrm{~mm}$ & 0.015 in & $0.381 \mathrm{~mm}$ & 0.015 in \\
\hline Fuel Element Length & $60.96 \mathrm{~cm}$ & $24.00 \mathrm{in}$ & $60.96 \mathrm{~cm}$ & $24.00 \mathrm{in}$ \\
\hline Fuel Composition & \multicolumn{2}{|c|}{$\mathrm{W}-60 \% \mathrm{UO}_{2}-6 \% \mathrm{Gd}_{2} \mathrm{O}_{3}$} & \multicolumn{2}{|c|}{ W $-60 \% \cup_{2}-6 \% \mathrm{Gd}_{2} \mathrm{O}_{3}$} \\
\hline Cladding Composition & \multicolumn{2}{|c|}{$\mathrm{W} / 25 \% \operatorname{Re}$} & \multicolumn{2}{|c|}{$\mathrm{W} / 25 \% \operatorname{Re}$} \\
\hline $\begin{array}{c}\text { Coolant Channels per } \\
\text { Element }\end{array}$ & \multicolumn{2}{|c|}{91} & \multicolumn{2}{|c|}{91} \\
\hline $\begin{array}{l}\text { Coolant Channel Diameter } \\
\text { without Cladding }\end{array}$ & $1.321 \mathrm{~mm}$ & 0.052 in & $1.524 \mathrm{~mm}$ & $0.060 \mathrm{in}$ \\
\hline $\begin{array}{l}\text { Coolant Channel Diameter } \\
\text { with Cladding }\end{array}$ & $0.914 \mathrm{~mm}$ & 0.036 in & $1.118 \mathrm{~mm}$ & $0.044 \mathrm{in}$ \\
\hline Coolant Channel Pitch & $2.353 \mathrm{~mm}$ & 0.0938 in & $2.353 \mathrm{~mm}$ & 0.0938 in \\
\hline
\end{tabular}




\section{Code Features and Capabilities}

- Developed by NASA, SAIC, and Westinghouse in early 1990's

- NESS is used for Conceptual Level Analysis of both the Reactor and Key Engine Subsystems

- NESS can Model Expander, Gas Generator, and Bleed Cycles

- GASPLUS for Liquid Hydrogen Properties

- Accept MCNP Analysis Results as Input

- Propellant Flow Rate Determined by Reactor Thermal Performance

- Able to Optimize Engine System Performance Based on Peak Allowable Fuel Temperature 


\section{NESS System Model}

- Reactor Component Mass and Thermal Energy Deposition Data from MCNP

- Fuel Elements and Tie-Tubes (NERVA Derived Systems Only)

- Control Drums, Radial Reflector, and Filler Elements

- Numerous Other Non-Nuclear Components

- Expander Cycle with Two Different Chamber Pressures

- 3.89 MPa (565 psia) for Criticality Limited Designs

- 6.89 MPa (1000 psia) for $111 \mathrm{kN}\left(25 \mathrm{klb}_{\mathrm{f}}\right.$ ) Class Engines

- Fixed Pump and Turbine Efficiency Values

- Pump Efficiency of $65 \%$

- Turbine Efficiency of $80 \%$

- Regeneratively Cooled Thrust Chamber and Nozzle to a Nozzle AR of 25:1

- Individually Orificed Fuel Elements

- Peak Allowable Fuel Temperature of 2860 K (5148 R)

- Total Nozzle Area Ratio 300:1

- RL10-B2 Style Retractable Nozzle Extension 


\section{NESS System Model (Cermet Only)}

- No Tie-Tubes, Periphery Fuel Elements Used to Provide Supplemental TPA Drive Energy as Required

- Added to TPA Drive Flow Path in Opposing Pairs

- No Longer Used to Heat Propellant to Produce Thrust

- Corresponding Fuel Element Pressure Drop Added to Cycle Flow Path

- Thermal Energy Added in Parallel Path to Control Drums and Radial Reflector

- Axial BeO Axial Reflector Treated

Computationally as Fuel Element Extension 


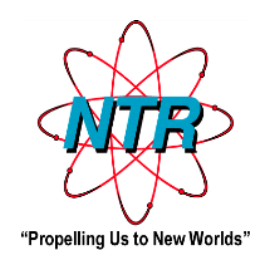

\section{NERVA Derived Engine Design Results}




\section{Normalized Thermal Energy Deposition Rate Profiles \\ NERVA Derived Engine Designs - Composite Fuel}

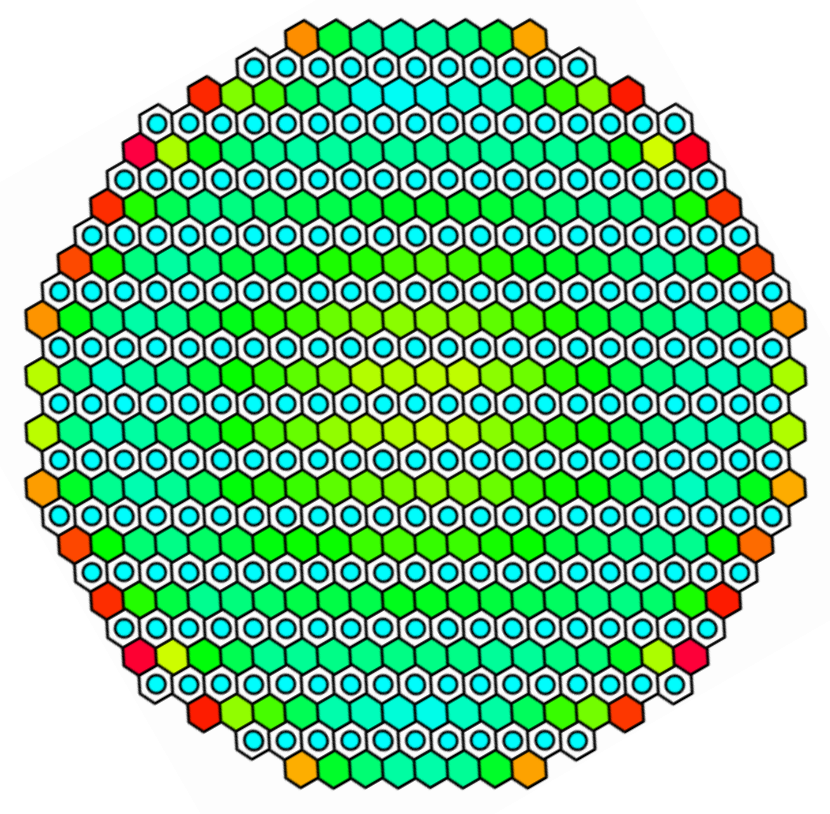

Criticality Limited NERVA Derived Option

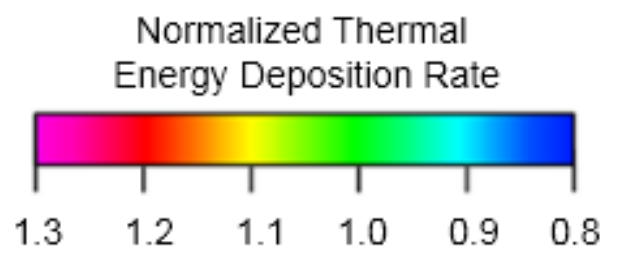

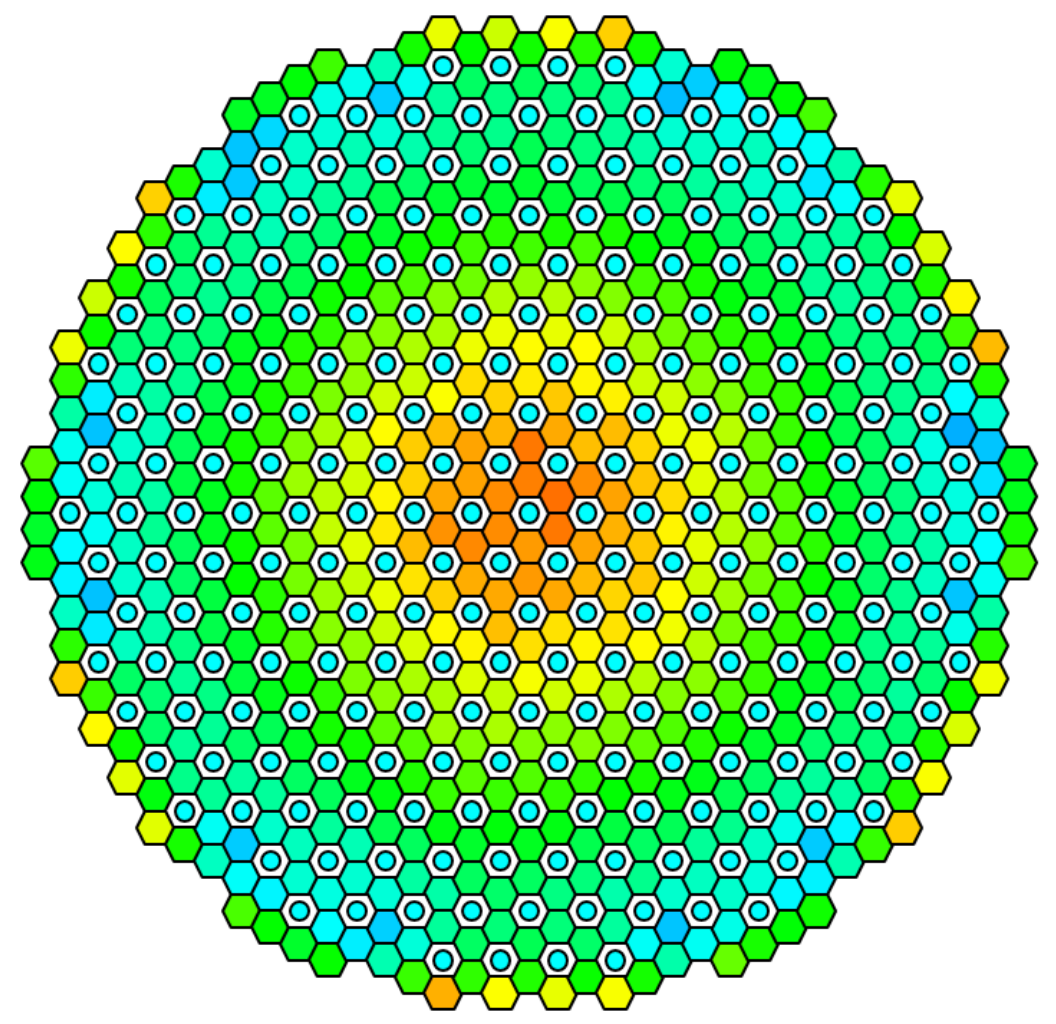

$111 \mathrm{kN}\left(25 \mathrm{klb}_{\mathrm{f}}\right)$ Thrust NERVA Derived Design

$\triangle$ Fuel Element

(O) Tie-Tube 


\section{Normalized Axial Thermal Energy Deposition Rate Profiles}

(NERVA Derived Designs)

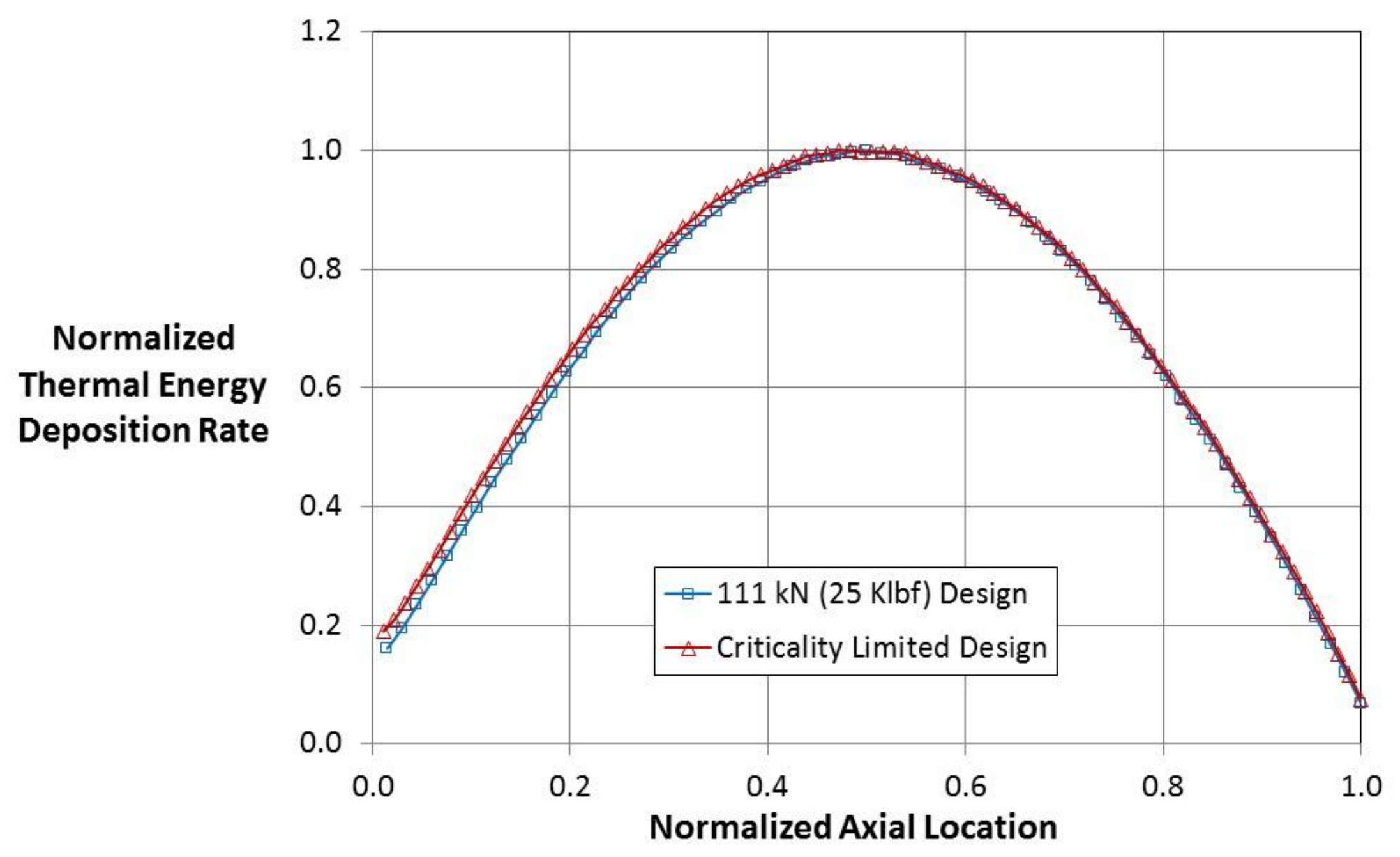




\section{NERVA Derived Expander Cycle Flow Path (Single TPA)}

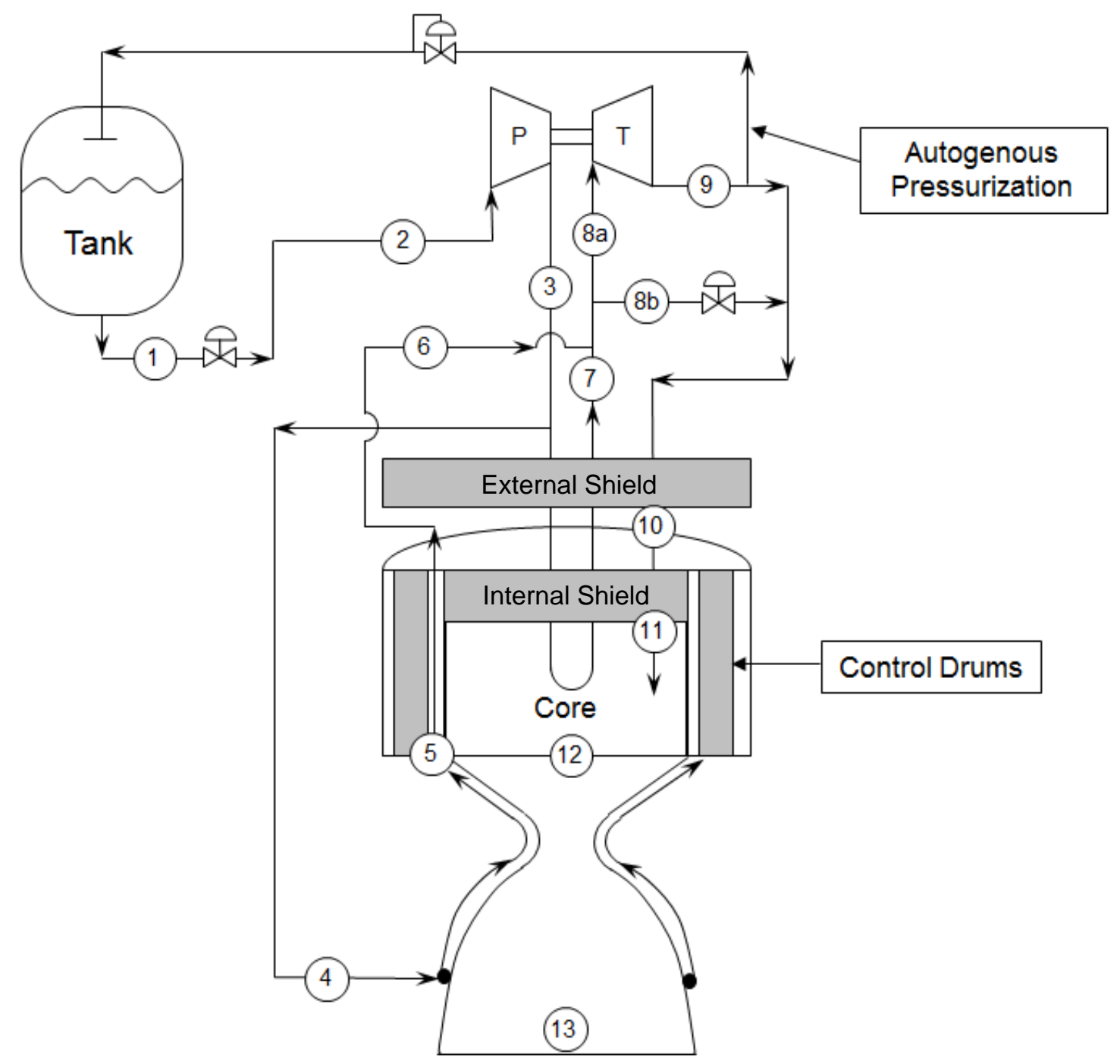




\section{NERVA Derived Engine Summary}

Peak Fuel Temperature of $\sim 2860 \mathrm{~K}$ and Nozzle Area Ratio (NAR) of $\sim 300: 1$

\begin{tabular}{|c|c|c|}
\hline & \multicolumn{2}{|c|}{ NERVA-Derived (Composite) } \\
\hline Masses $(\mathrm{kg})$ & Criticality-Limited & $25 \mathrm{klb}_{\mathrm{f}}$ Class \\
\hline Fuel Elements (FE) & 207.7 & 612.84 \\
\hline Tie Tubes (TT) & 231.0 & 313.70 \\
\hline Heater Elements (HE) & - & - \\
\hline Reflector Assembly & 717.71 & 1141.57 \\
\hline Pressure Vessel & 87.93 & 284.72 \\
\hline TPA & 9.07 & 41.43 \\
\hline TVC, Lines, and Valves & 38.2 & 85.82 \\
\hline Nozzle & 81.03 & 149.78 \\
\hline Assorted Hardware & 416.36 & 708.14 \\
\hline Engine Mass & 1789 & 3338 \\
\hline \multicolumn{3}{|l|}{ Dimensions (cm) } \\
\hline Core / FE Length & 89 & 132 \\
\hline TPA / TVC System Length & 178.1 & 228.3 \\
\hline Pressure Vessel Length & 207.1 & 320.9 \\
\hline Nozzle Length & 233.7 & 320.2 \\
\hline Nozzle Exit Diameter & 137.9 & 189.0 \\
\hline Approx. Total Engine Length & 618.9 & 869.4 \\
\hline \multicolumn{3}{|l|}{ Engine Parameters } \\
\hline No. Elements (FE/TT/HE) & $260 / 251 / 0$ & $564 / 241 / 0$ \\
\hline Core Power Level $\left(\mathrm{MW}_{\mathrm{t}}\right)$ & 157 & 563 \\
\hline Chamber Pressure (MPa) & 3.89 & 6.89 \\
\hline U-235 Mass $(\mathrm{kg})$ & 27.5 & 36.8 \\
\hline Thrust $\left(k \mid b_{f}\right)$ & 7.52 & 25.18 \\
\hline Thrust-to-Weight Ratio & 1.91 & 3.42 \\
\hline Delivered Isp (s) & 894 & 909 \\
\hline
\end{tabular}




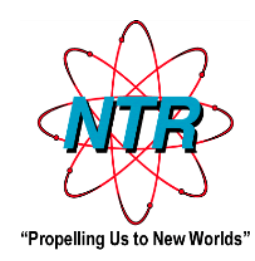

\section{Cermet Engine Design Results}

Glenn Research Center 


\section{Normalized Thermal Energy Deposition Rate Profiles Cermet Fuel Based Engine Designs}

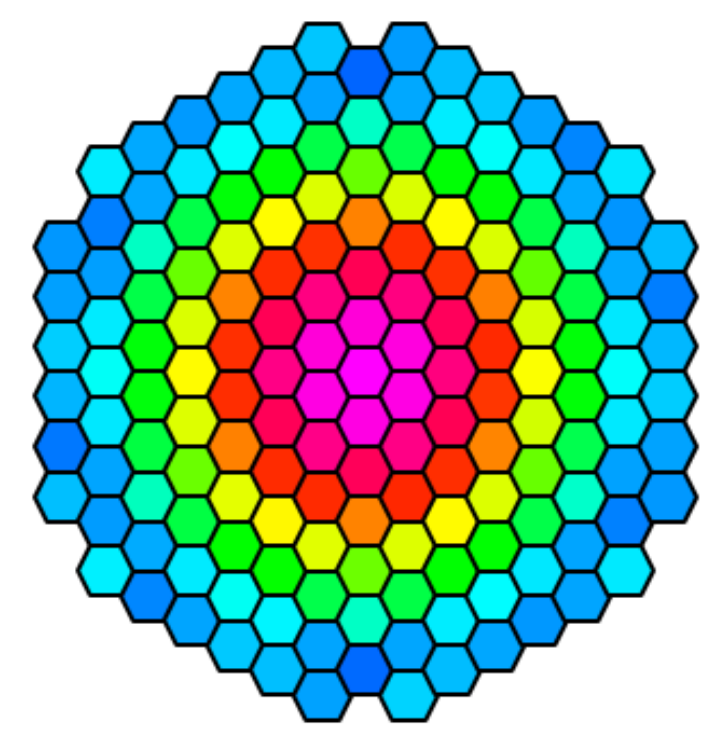

Criticality Limited ANL-200 Based Design

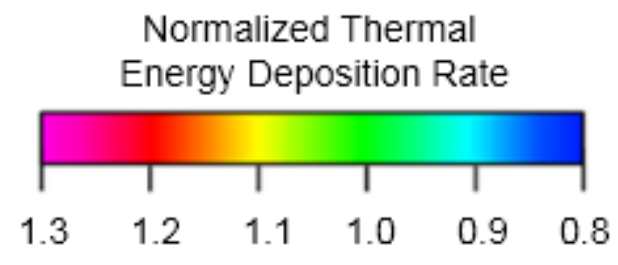

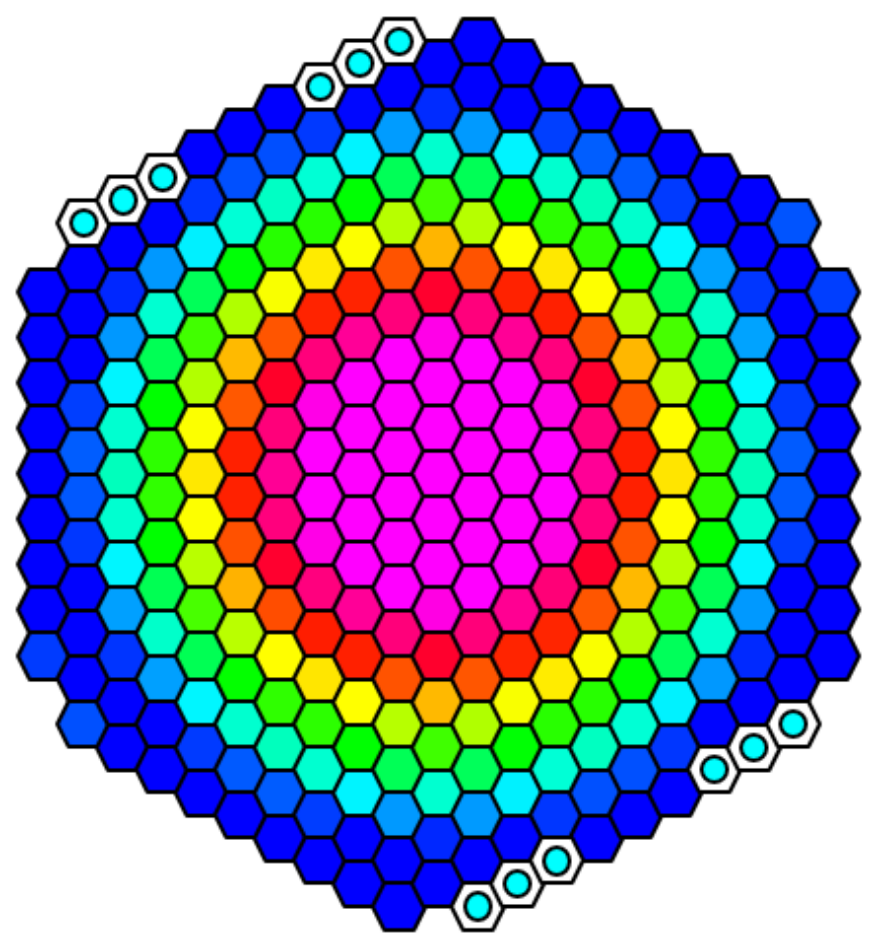

$111 \mathrm{kN}\left(25 \mathrm{klb}_{\mathrm{f}}\right)$ Thrust GE-711 Based Design

\footnotetext{
$\square$ Fuel Element

(O) Driver Element
} 


\section{Normalized Axial Thermal Energy Deposition Rate Profiles}

(Cermet Designs)

Normalized

Thermal Energy 0.60 Deposition Rate

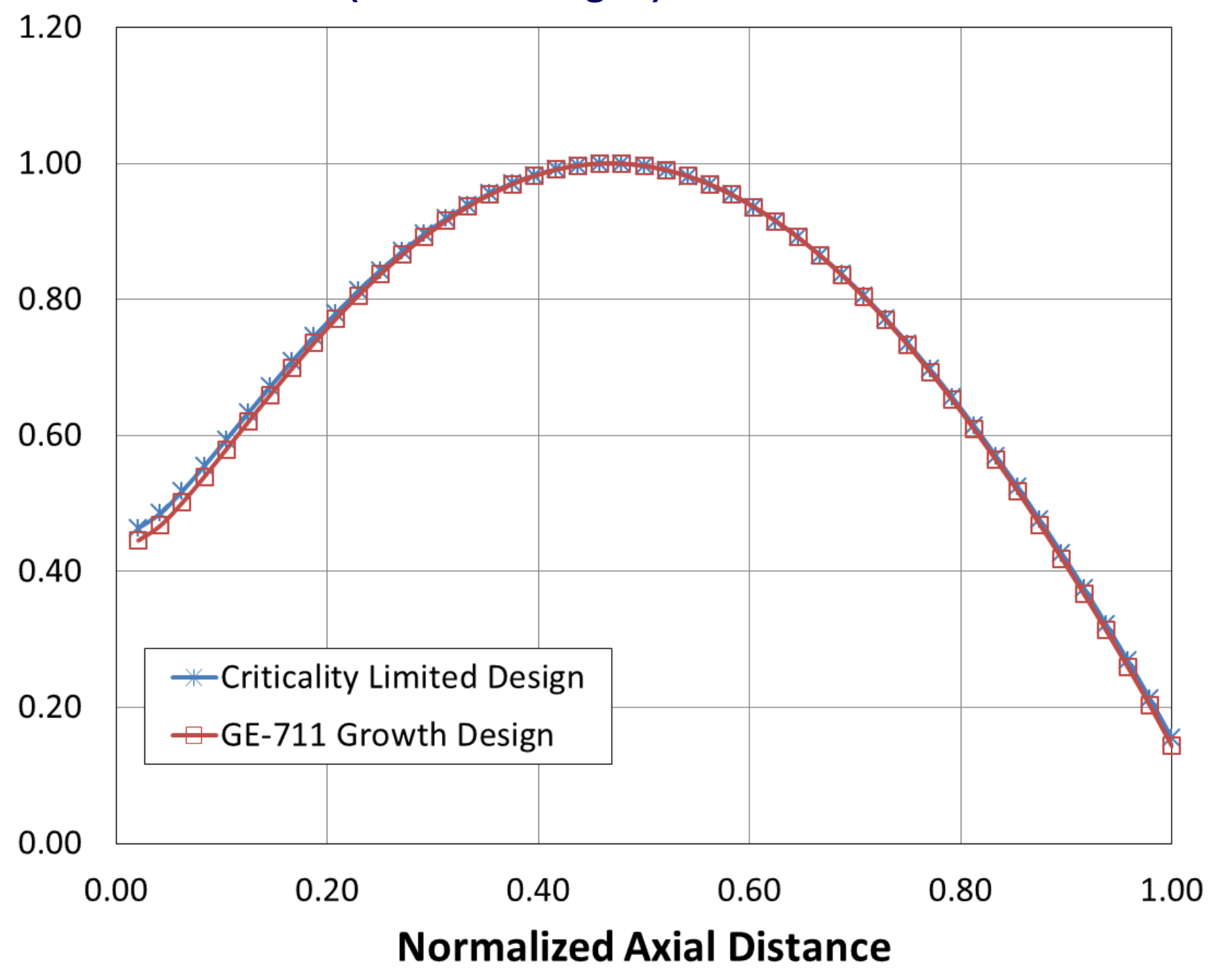




\section{Cermet Based NTR Flow Diagram}

\section{(Expander Cycle with Single TPA)}

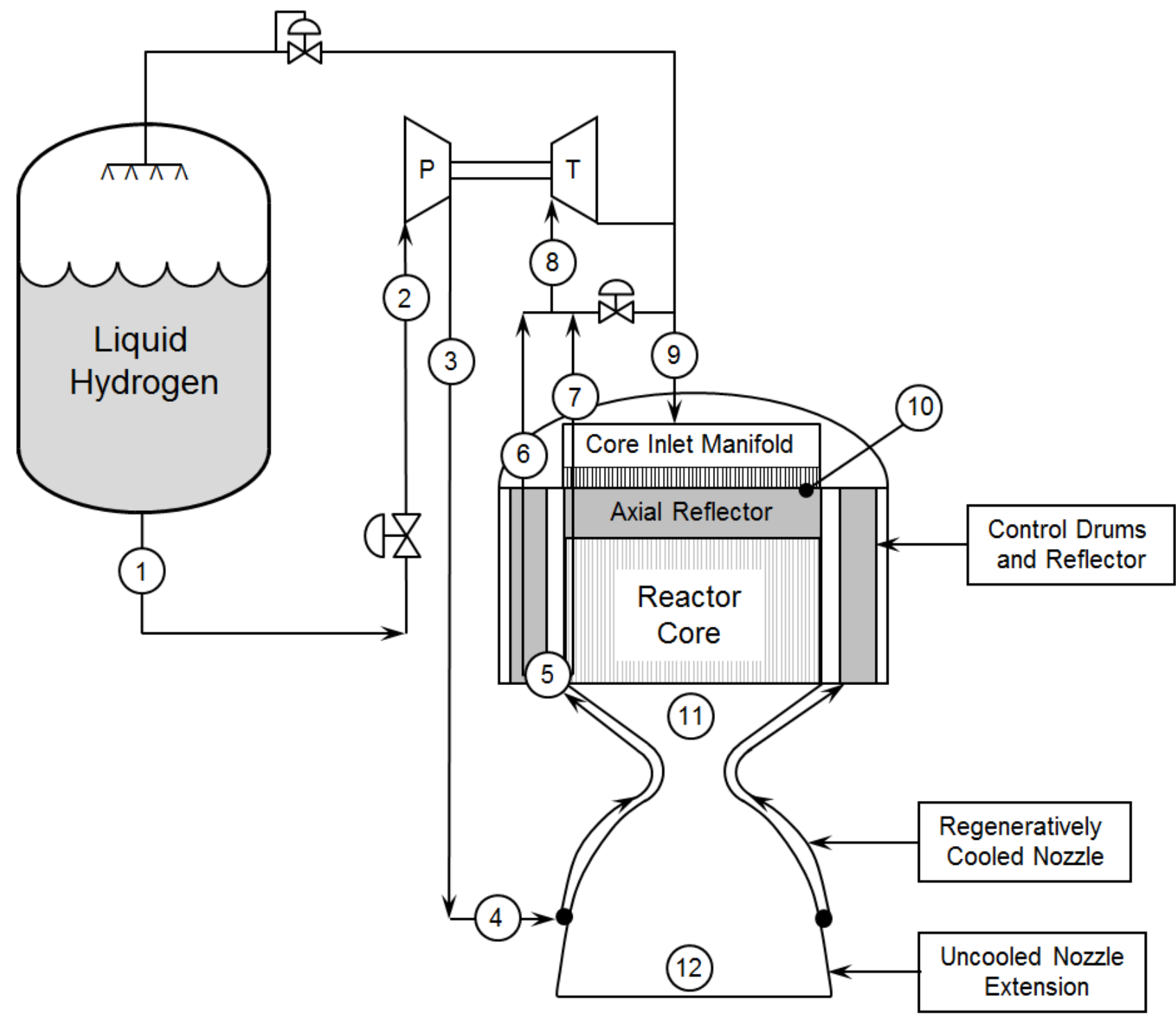




\section{Point of Departure Engine Summary}

Peak Fuel Temperature of $\sim 2860 \mathrm{~K}$ and Nozzle Area Ratio (NAR) of $\sim 300: 1$

\begin{tabular}{|c|c|c|c|c|}
\hline & \multicolumn{2}{|c|}{ NERVA-Derived (Composite) } & \multirow{2}{*}{$\begin{array}{c}\text { ANL-200 } \\
\text { Criticality-Limited }\end{array}$} & \multirow{2}{*}{$\begin{array}{l}\text { GE-711 Variant } \\
25 \mathrm{klb}_{\mathrm{f}} \text { Class }\end{array}$} \\
\hline Masses (kg) & Criticality-Limited & $25 \mathrm{klb}_{\mathrm{f}}$ Class & & \\
\hline Fuel Elements (FE) & 207.7 & 612.84 & 950.50 & 1226.63 \\
\hline Tie Tubes (TT) & 231.0 & 313.70 & - & - \\
\hline Heater Elements (HE) & - & - & - & 48.90 \\
\hline Reflector Assembly & 717.71 & 1141.57 & 414.74 & 437.51 \\
\hline Pressure Vessel & 87.93 & 284.72 & 74.98 & 205.28 \\
\hline TPA & 9.07 & 41.43 & 13.28 & 53.04 \\
\hline TVC, Lines, and Valves & 38.2 & 85.82 & 42.68 & 73.39 \\
\hline Nozzle & 81.03 & 149.78 & 106.95 & 149.49 \\
\hline Assorted Hardware & 416.36 & 708.14 & 208.87 & 480.76 \\
\hline Engine Mass & 1789 & 3338 & 1812 & 2675 \\
\hline \multicolumn{5}{|l|}{ Dimensions (cm) } \\
\hline Core / FE Length & 89 & 132 & 71 & 61 \\
\hline TPA / TVC System Length & 178.1 & 228.3 & 209.3 & 225.3 \\
\hline Pressure Vessel Length & 207.1 & 320.9 & 155.0 & 157.0 \\
\hline Nozzle Length & 233.7 & 320.2 & 292.0 & 318.9 \\
\hline Nozzle Exit Diameter & 137.9 & 189.0 & 172.3 & 188.2 \\
\hline Approx. Total Engine Length & 618.9 & 869.4 & 656.3 & 701.2 \\
\hline \multicolumn{5}{|l|}{ Engine Parameters } \\
\hline No. Elements (FE/TT/HE) & $260 / 251 / 0$ & 564 / $241 / 0$ & $163 / 0 / 0$ & $313 / 0 / 12$ \\
\hline Core Power Level $\left(\mathrm{MW}_{\mathrm{t}}\right)$ & 157 & 563 & 266 & 564 \\
\hline Chamber Pressure (MPa) & 3.89 & 6.89 & 3.89 & 6.89 \\
\hline U-235 Mass $(\mathrm{kg})$ & 27.5 & 36.8 & 238.5 & 258.6 \\
\hline Thrust $\left(k \mid b_{f}\right)$ & 7.52 & 25.18 & 11.92 & 25.09 \\
\hline Thrust-to-Weight Ratio & 1.91 & 3.42 & 2.98 & 4.26 \\
\hline Delivered Isp (s) & 894 & 909 & 903 & 906 \\
\hline
\end{tabular}




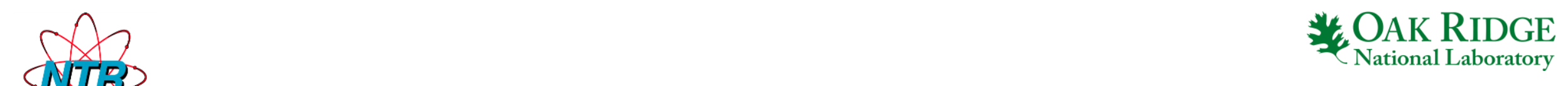

\section{Conclusions}

- Four Revised Point of Departure NTR Engines were Designed and Analyzed using MCNP and NESS

- All Four Engines Have Thermodynamically Closed Cycles at Nominal Chamber Pressures

- $111 \mathrm{kN}\left(25 \mathrm{klb}_{\mathrm{f}}\right)$ Cermet Design Required Dedicated Heater Elements to Close the Cycle

- Cermet Based Designs had Slightly Higher T/W Ratios, but Required Substantially More U-235

- NERVA Derived Criticality Limited Engine Could Operate at Lower Power and Thrust Levels Compared to the Criticality Limited Cermet Design 


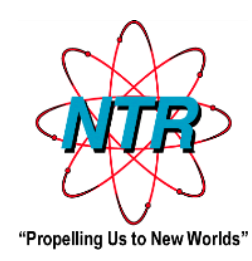

\section{Back-up Charts}




\section{Carbide/Graphite Based Fuels}

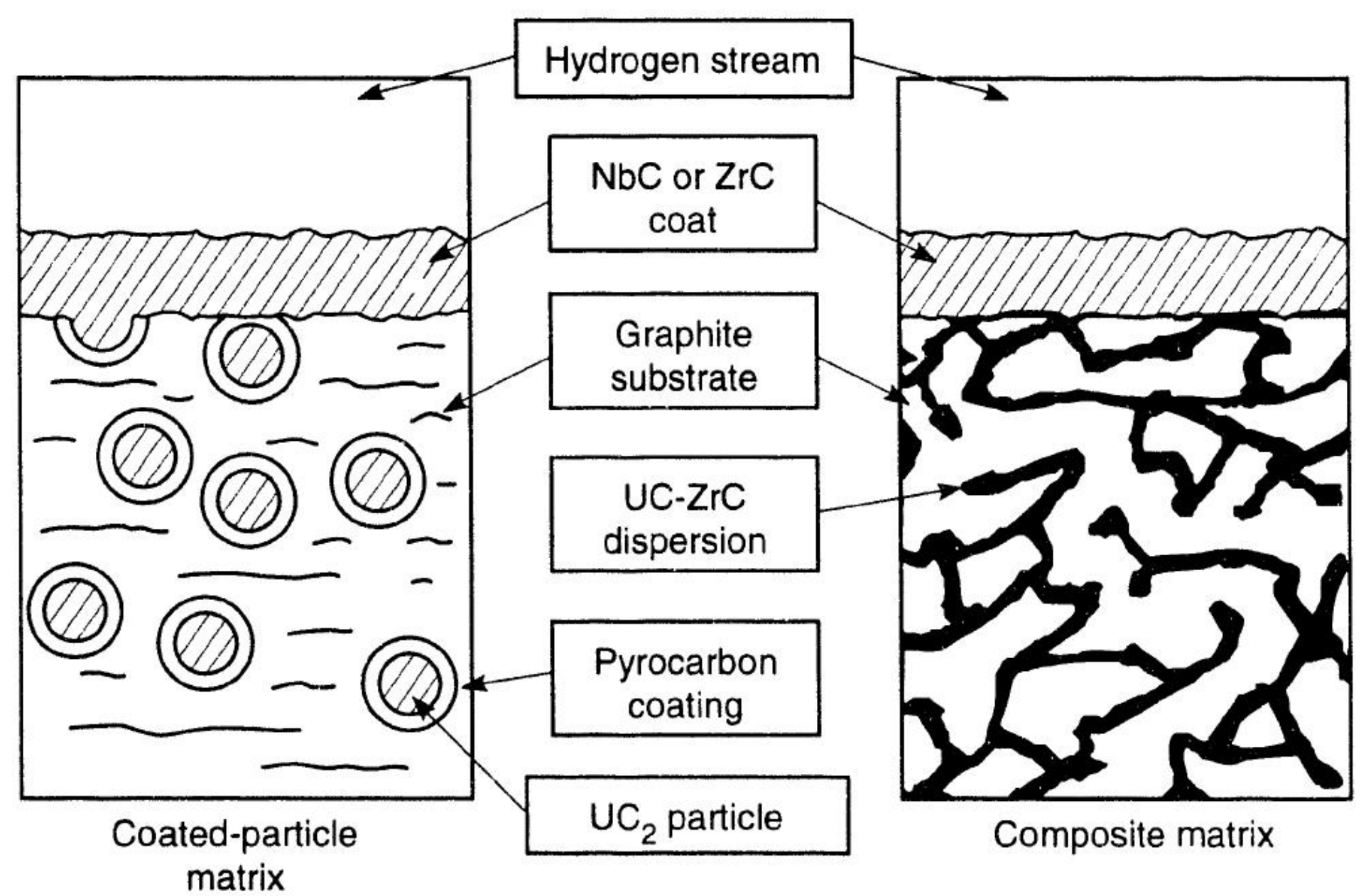

Glenn Research Center 


\section{RL10-B2 Retractable Nozzle}

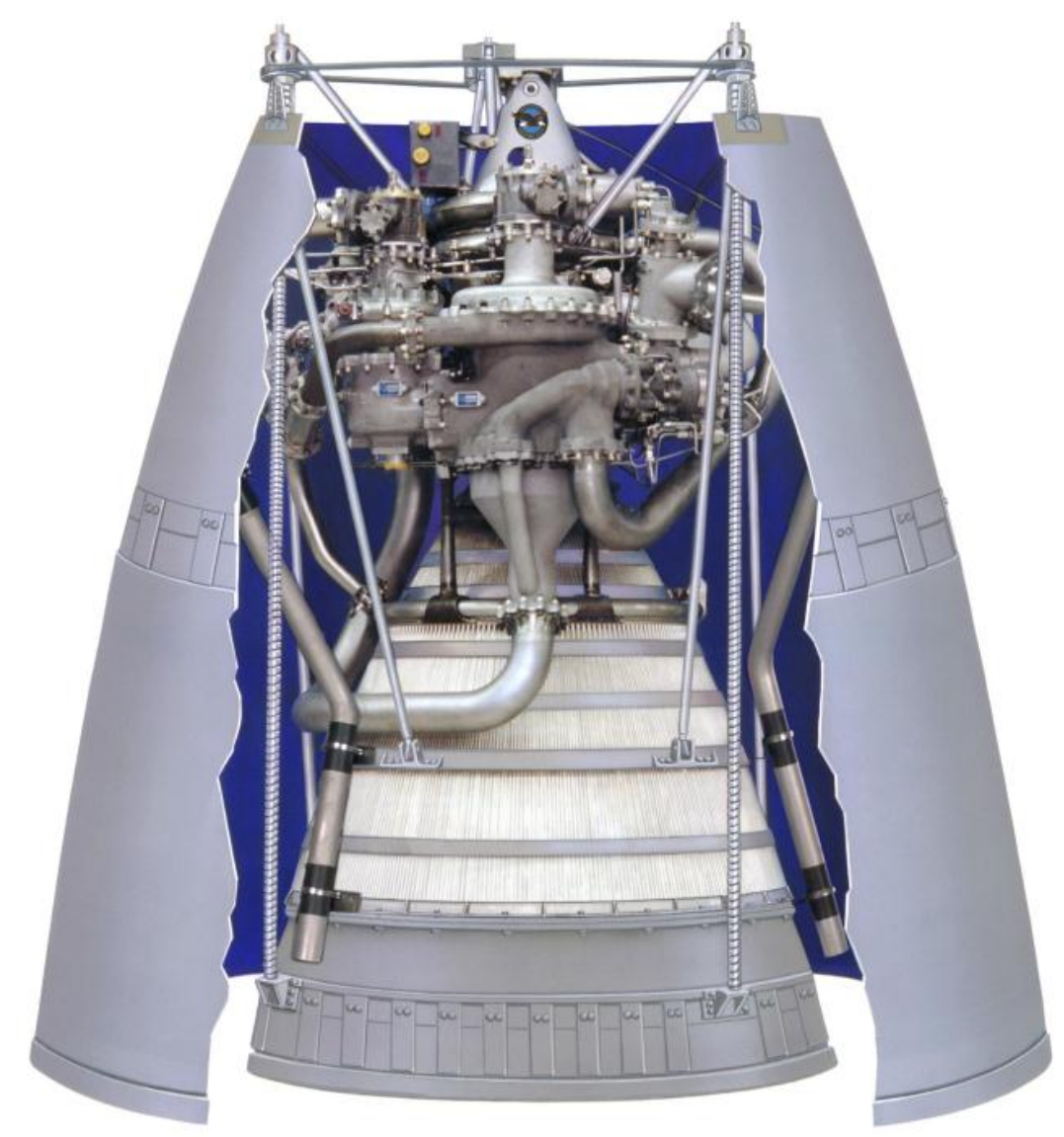

Nozzle Extension Retracted

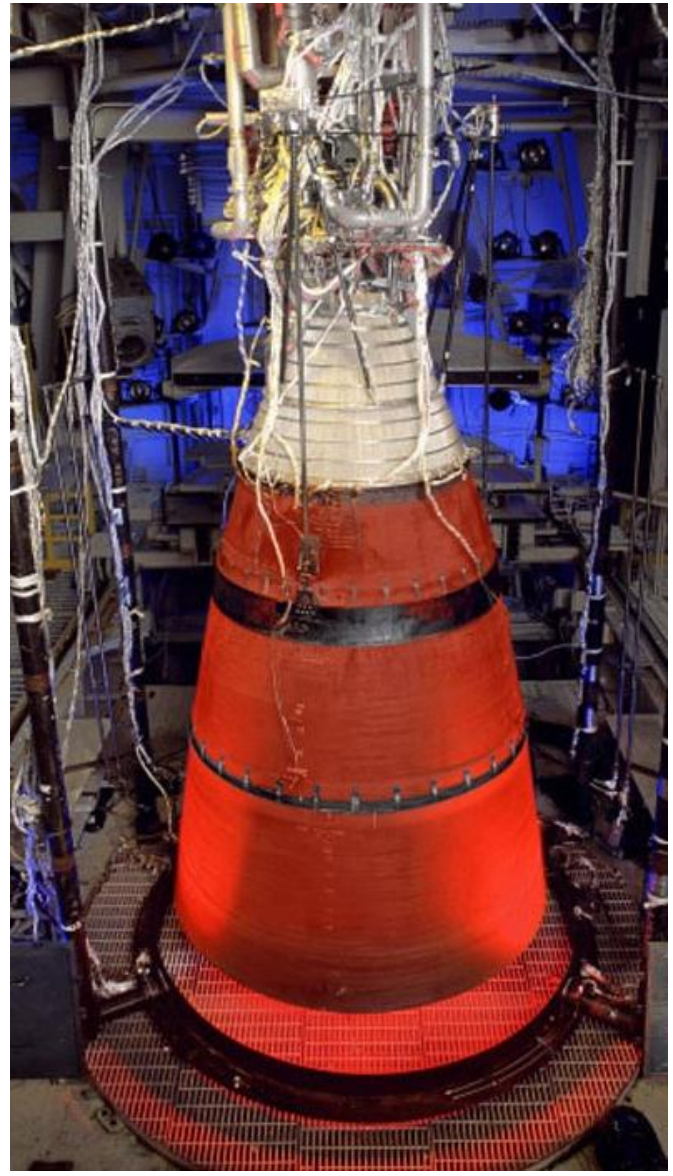

Nozzle Extension Deployed 


\section{Deployable Nozzle Components}

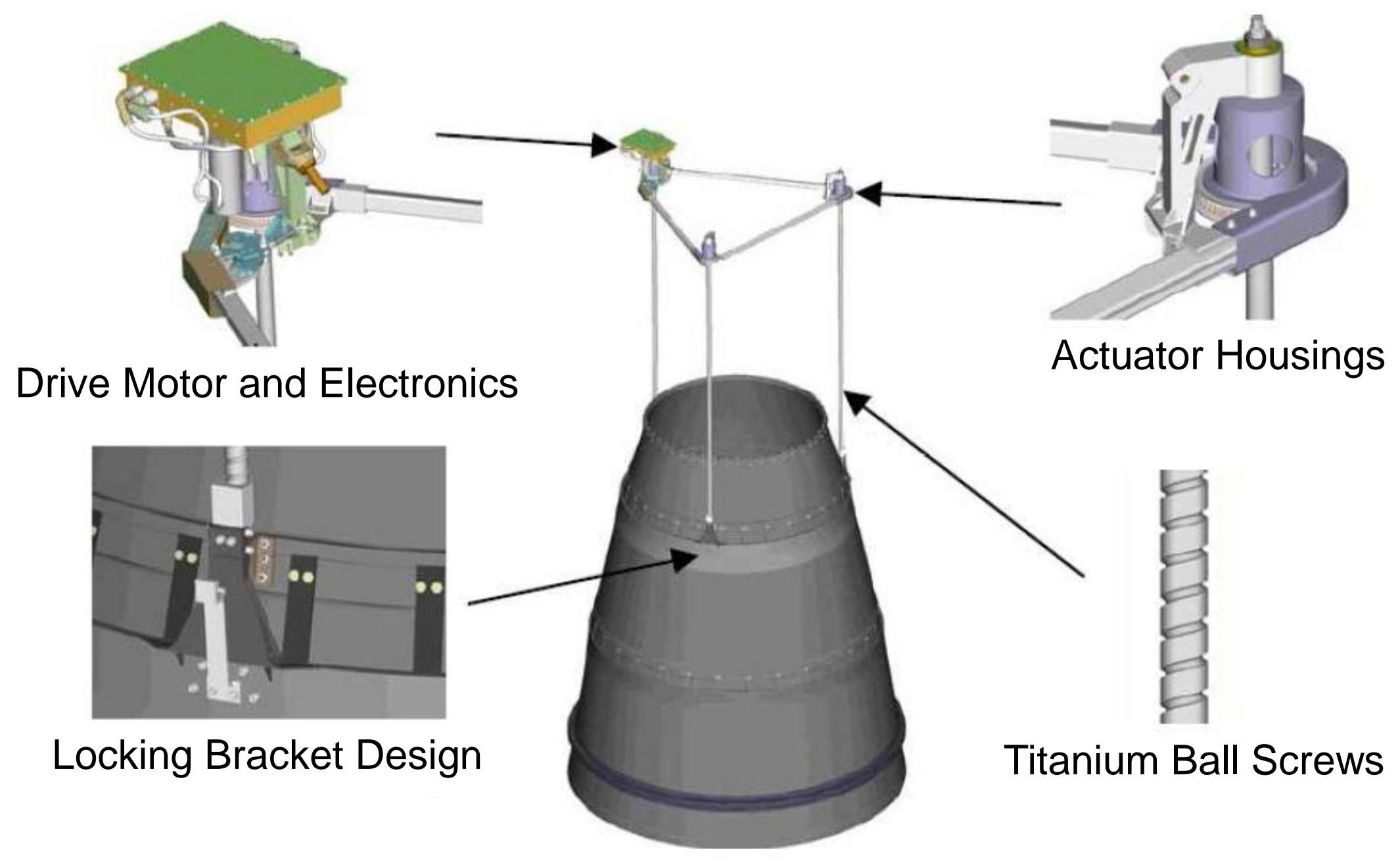




\section{Translating Nozzle Bracket Design}

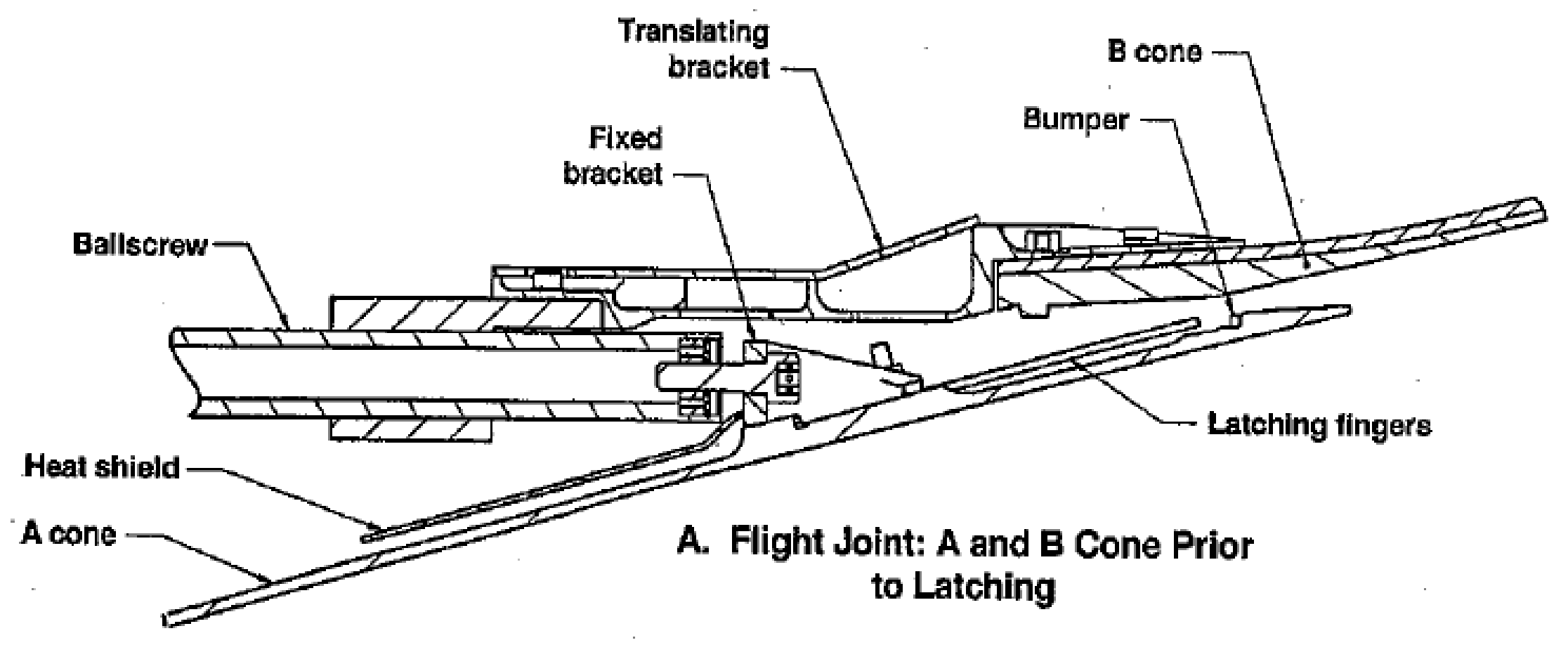

Glenn Research Center 


\section{Translating Nozzle Bracket, cont.}

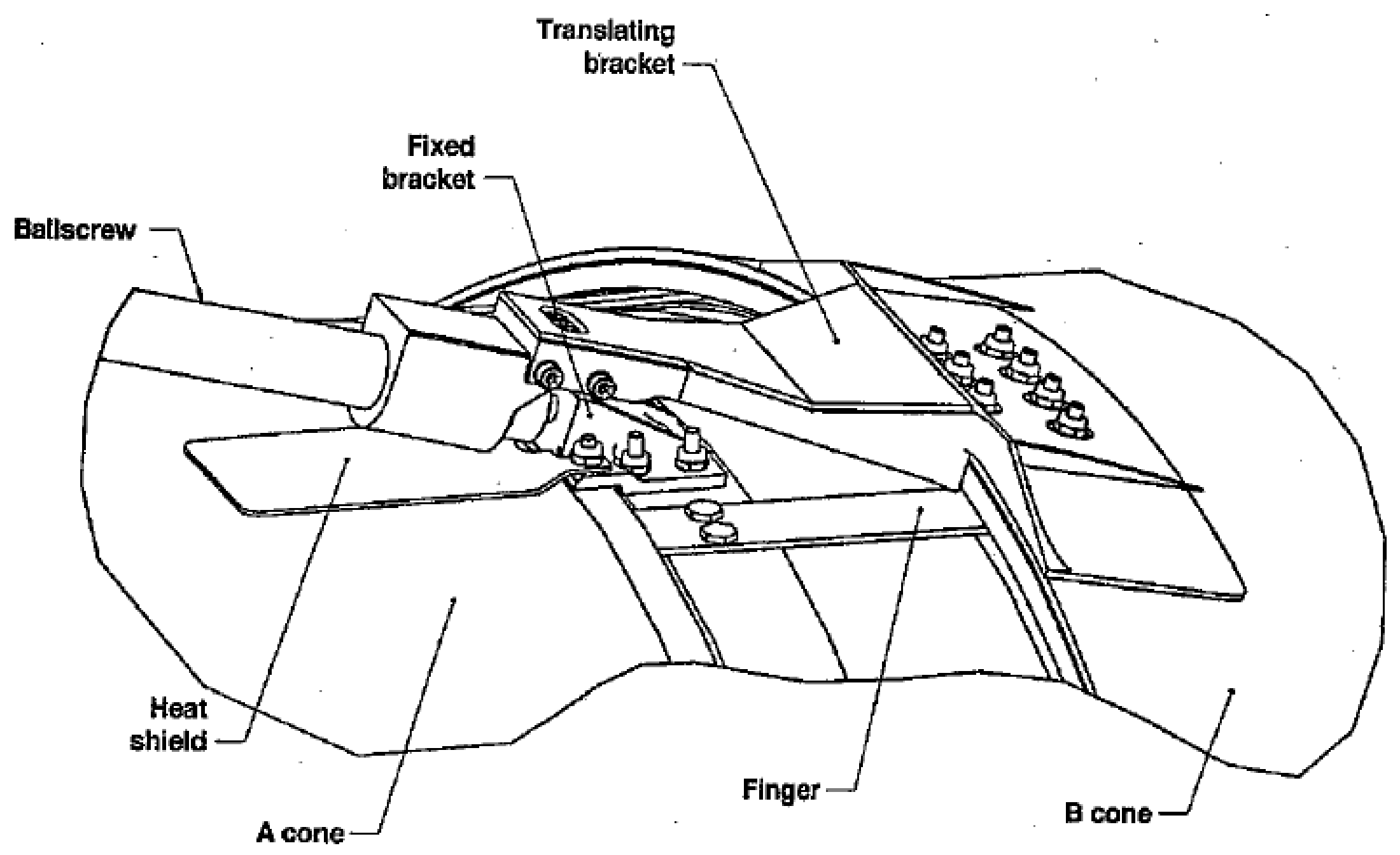

Glenn Research Center 


\begin{tabular}{|c|c|c|c|c|c|c|c|}
\hline \multirow{2}{*}{ Station } & \multirow{2}{*}{ No. } & \multicolumn{2}{|c|}{ Flow Rate } & \multicolumn{2}{|c|}{ Pressure } & \multicolumn{2}{|c|}{ Temperature } \\
\hline & & $(\mathrm{lbm} / \mathrm{s})$ & $(\mathrm{kg} / \mathrm{s})$ & (psia) & (MPa) & (R) & (K) \\
\hline Tank Exit & 1 & 8.50 & 3.86 & 28.20 & 0.194 & 30.6 & 17.0 \\
\hline Pump Inlet & 2 & 8.50 & 3.86 & 18.28 & 0.126 & 30.6 & 17.0 \\
\hline Pump Exit & 3 & 8.50 & 3.86 & 976.0 & 6.728 & 44.17 & 24.54 \\
\hline Tie Tube and Slat Inlet & 3 & 4.65 & 2.11 & 940.1 & 6.481 & 44.17 & 24.54 \\
\hline Tie Tubeand Slat Exit & 7 & 4.65 & 2.11 & 840.1 & 5.791 & 749.6 & 416.5 \\
\hline Nozzle Inlet & 4 & 3.86 & 1.75 & 940.1 & 6.481 & 44.17 & 24.54 \\
\hline Reflector Inlet & 5 & 3.86 & 1.75 & 865.1 & 5.964 & 438.4 & 243.5 \\
\hline Reflector Exit & 6 & 3.86 & 1.75 & 840.1 & 5.791 & 530.2 & 294.5 \\
\hline Turbine Inlet & $8 a$ & 7.50 & 3.40 & 840.1 & 5.791 & 677.5 & 376.4 \\
\hline Turbine Bypass & $8 b$ & 1.00 & 0.45 & 840.1 & 5.791 & 677.5 & 376.4 \\
\hline Turbine Exit & 9 & 7.50 & 3.40 & 717.0 & 4.943 & 644.5 & 358.1 \\
\hline Reactor Inlet & 10 & 8.45 & 3.84 & 667.3 & 4.600 & 644.5 & 358.1 \\
\hline Fuel Element Inlet & 11 & 8.45 & 3.84 & 641.9 & 4.425 & 645.8 & 358.8 \\
\hline Chamber Inlet & 12 & 8.45 & 3.84 & 565.0 & 3.895 & 4922.7 & 2734.8 \\
\hline Nozzle Exit (Static) & 13 & 8.45 & 3.84 & 0.033 & 0.0002 & 196.5 & 109.2 \\
\hline
\end{tabular}

NOTE: Engine Fuel Element length is $89 \mathrm{~cm} / 35$ inches and uses single TPA 


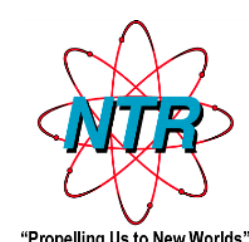

\section{Relative NTR Engine Size}

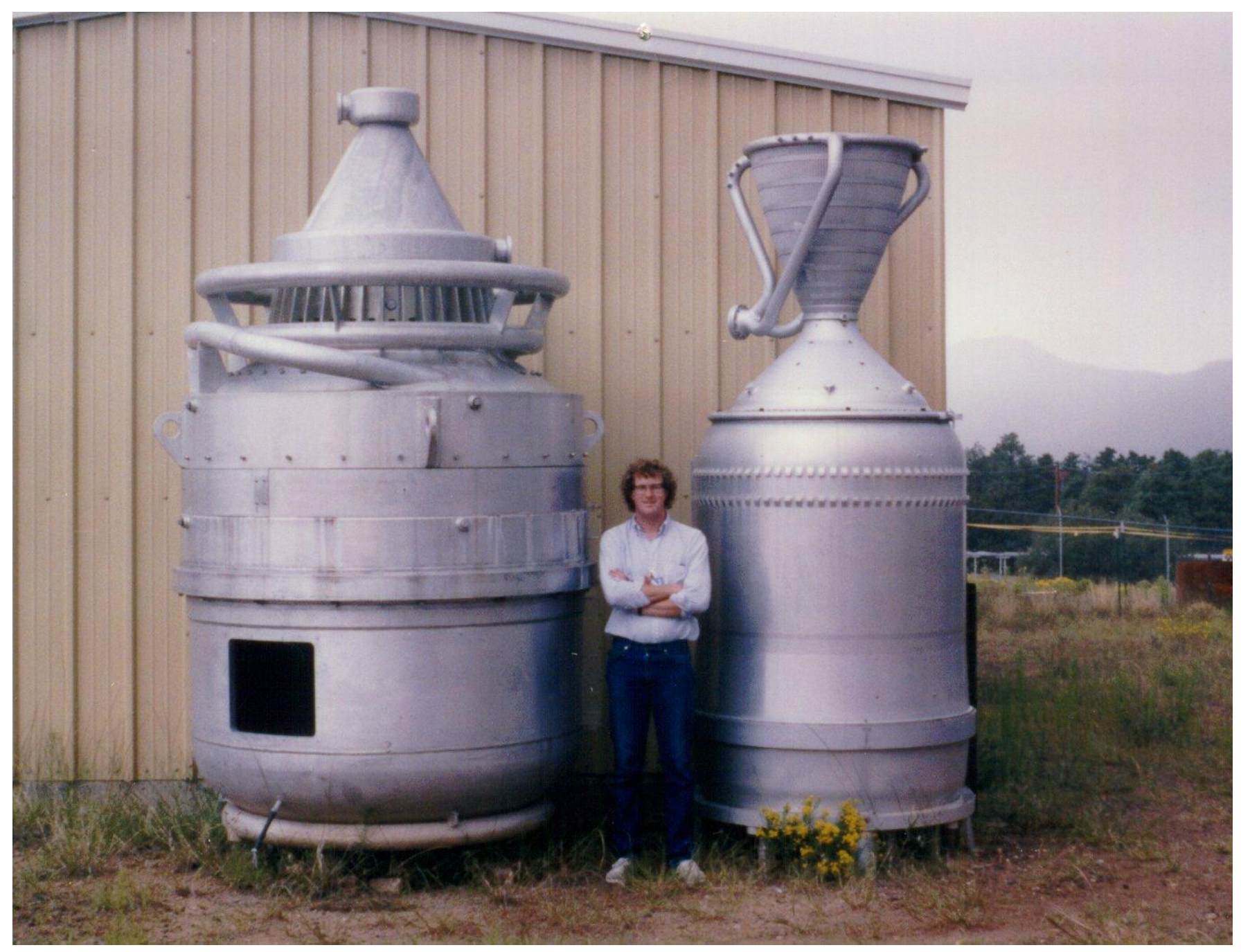

Glenn Research Center 


\section{Hydrogen Dissociation}

Potential Performance with Hydrogen Dissociation

- $\quad \mathrm{ISP} \sim(\mathrm{Tc} / \mathrm{Mw})^{\wedge} 0.5$

- Potential Performance Increase with Hydrogen Dissociation

- Lower Pressure and Higher Temperature Allow for Dissociation

- NTR System Size and Mass Tend to Increase with Lower Pc

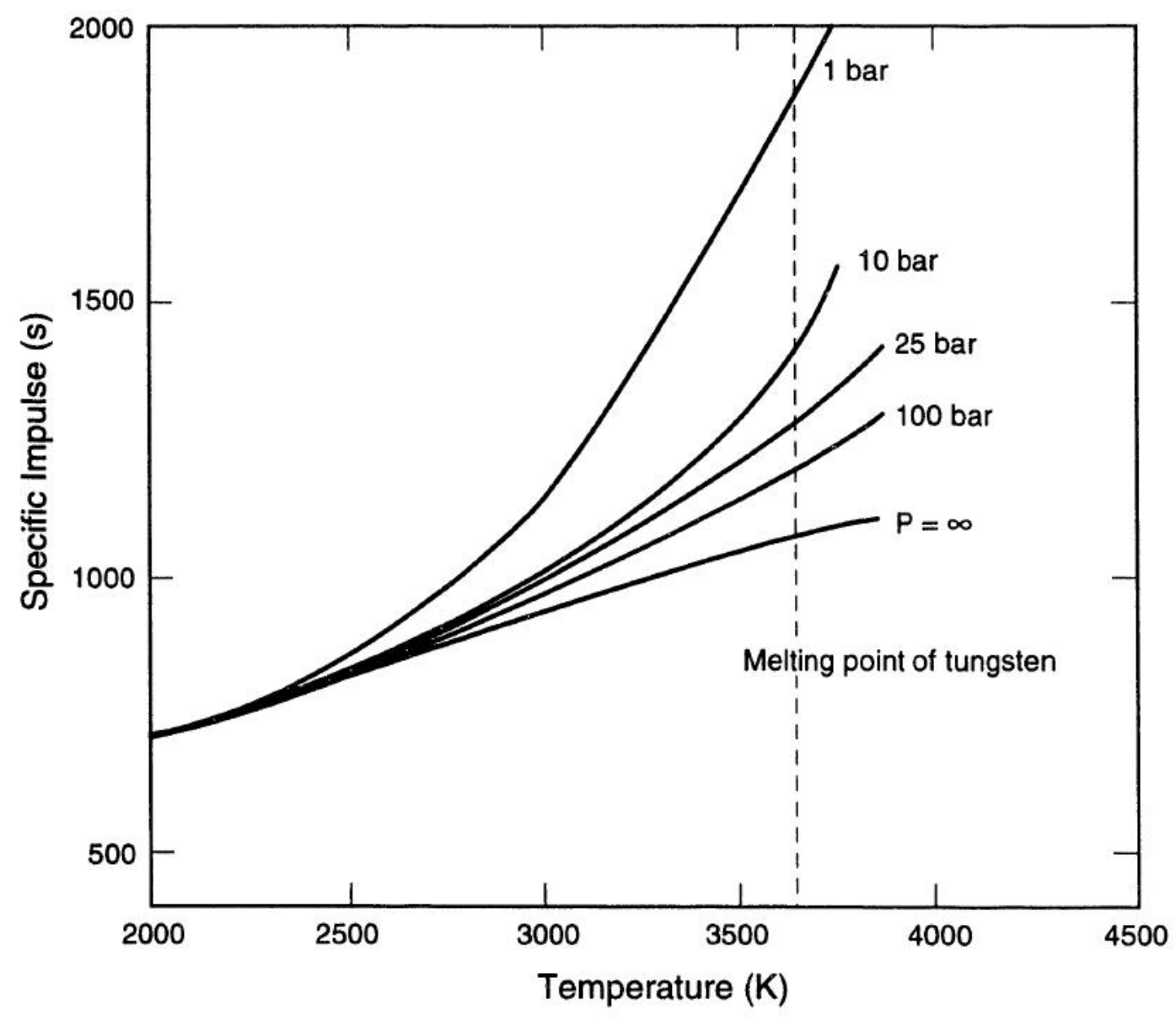




\section{Hydrogen Propellant}

- Hydrogen Delivers Highest Possible ISP for Given Tch, Pch, and Nozzle AR

- Current and Well Understood Technology

- Allows for Simpler and more Robust Design if used as Monopropellant

- Dissociation at Lower Pch Yields Even Higher ISP

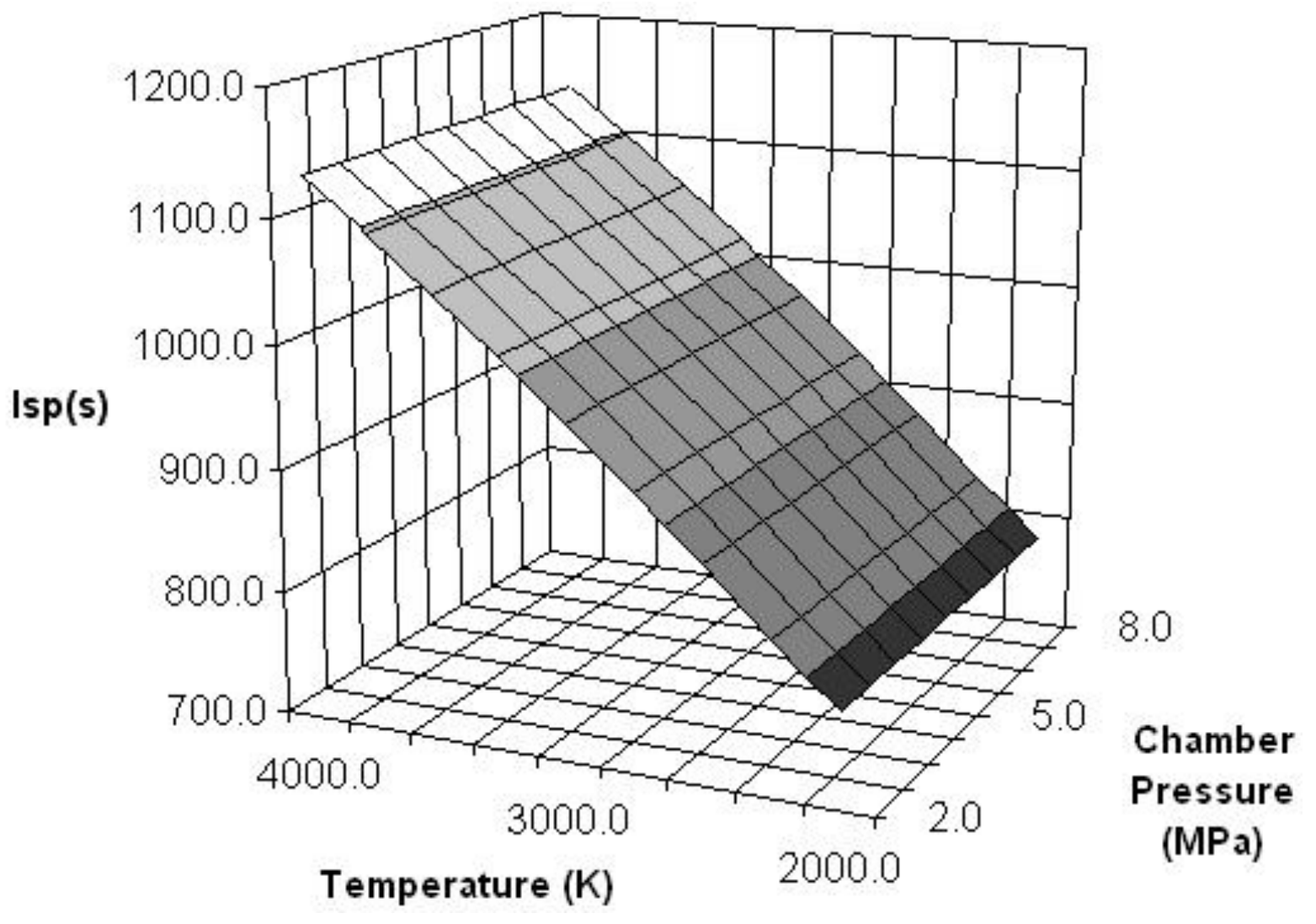

Hydrogen Propellant Performance (300:1 Nozzle Area Ratio, Isentropic Expansion, Ionized Species) 